\title{
COVARIANTS OF POWER SERIES
}

\author{
By J. E. Wright.
}

[Received October 6th, 1904.-Read November 10th, 1904.]

1. The theory of perpetuants in the symbols of Aronhold has been dealt with in several recent papers. In the present it is proposed to consider perpetuants when the form or: forms of infinite order are taken to be power series." "This is of course the natural interpretation.

The covariant $(a b)^{\lambda} a_{x}^{n-\lambda} b_{x}^{n-\lambda}$ of the forms $a_{x}^{n}, b_{x}^{n}$ may be represented simply as $(a b)^{\lambda}$ when $n$ is infinite. In addition, the variable $x_{1}$ is replaced by unity, as we are concerned with ordinary power series.

Now $\quad x_{1}^{\lambda}(a b)^{\lambda}=\left(a_{x} b_{2}-a_{2} b_{x}\right)^{\lambda}$.

If we expand the expression on the right, we have

$$
x_{1}^{\lambda}(a b)^{\lambda}=a_{x}^{\lambda} b_{2}^{\lambda}-\lambda a_{x}^{\lambda-1} b_{2}^{\lambda-1} a_{2} b_{x}+\ldots+(-1)^{\lambda} a_{2}^{\lambda} b_{x}^{\lambda} .
$$

Also

$$
\left(\frac{\partial}{\partial x_{2}}\right)^{\mu} b_{x}^{n}=n(n-1) \ldots(n-\mu+1) b_{2}^{\mu} b_{x}^{n-\mu}
$$

and therefore

$$
b_{2}^{\mu} b_{x}^{\lambda-\mu}=\frac{1}{n(n-1) \ldots(n-\mu+1)} \frac{1}{b_{x}^{n-\lambda}}\left(\frac{\partial}{\partial x_{2}}\right)^{\mu} b_{x}^{n} .
$$

If in this we make $n$ very large, we have practically

$$
b_{2}^{\mu} b_{x}^{\lambda-\mu}=\frac{1}{n^{\mu}} \frac{1}{b_{x}^{n}}\left(\frac{\partial}{\partial x_{2}}\right)^{\mu} b_{x}^{n} .
$$

Calling $a_{x}^{n}, b_{x}^{n}, f(x), \phi(x)$ respectively, and omitting the suffix 2 from $x_{2}$, we have, finally,

$$
\begin{aligned}
x_{1}^{\lambda}(a b)^{\lambda} & =\frac{1}{n^{\lambda}}\left\{f \frac{\partial^{\lambda} \phi}{\partial x^{\lambda}}-\lambda \frac{\partial J}{\partial x} \frac{\partial^{\lambda-1} \phi}{\partial x^{\lambda-1}}+\ldots+(-1)^{\lambda} \phi \frac{\partial^{\lambda} f}{\partial x^{\lambda}}\right\} \\
& =\frac{1}{n^{\lambda}}\left(\frac{\partial}{\partial x}-\frac{\partial}{\partial y}\right)^{\lambda} f(x) \phi(y),
\end{aligned}
$$

- Acknowledgment ought to be made of the writer's indebtedness to Mr. J. H. Grace. The results proved in this paper in accordance with which the vanishing of certain covariants of power series marks the functions represented by the series as exponential or elliptic were communicated by Mr. Grace to the Society in June, 1904, and were placed by him at the writer's disposal. 
where $y$ is replaced by $x$ after differentiation. This shows that the perpetuant $(a b)^{\lambda}$ of the forms $f(x)$ and $\phi(x)$ is, for all practical purposes,

$$
\left(\frac{\partial}{\partial x}-\frac{\partial}{\partial y}\right)^{\lambda} f(x) \phi(y) \quad(y=x)
$$

Exactly similarly, the perpetuant $(b c)^{\lambda}(c a)^{\mu}(a b)^{\nu}$ of three forms $f, \phi, \psi$ may be taken to be

$$
\left(\frac{\partial}{\partial y}-\frac{\partial}{\partial z}\right)^{\lambda}\left(\frac{\partial}{\partial z}-\frac{\partial}{\partial x}\right)^{\mu}\left(\frac{\partial}{\partial x}-\frac{\partial}{\partial y}\right)^{\nu} f(x) \phi(y) \psi(z) \quad(y=z=x),
$$

and the generalisation is obvious.

Incidentally this shows that in the appropriate symbols of MacMahon, wherein $f=e^{a x}$, and $\alpha$ is a symbol, the covariants are of the type $(\alpha-\beta)^{\lambda}, \quad(\beta-\gamma)^{\lambda}(\gamma-\alpha)^{\mu}(\alpha-\beta)^{\nu}$, and so on ; and, further, that the rules for writing down complete systems are exactly the same as in the ordinary Aronhold symbols.

2. The method for calculating covariants of a power series shows at once that all the covariants have the same circle of convergence as the original form. In the particular cases considered below the forms converge over the whole of the finite part of the plane.

Consider first the exponential series. We have only one form $f \equiv e^{a x}$, and the Hessian is

$$
\left(\frac{\partial}{\partial x}-\frac{\partial}{\partial y}\right)^{2} e^{a x} e^{a y}=0 \quad(y=x)
$$

and, in like manner, every other covariant vanishes. This result is to be expected, for every covariant of a perfect $n$-th power vanishes, and the exponential function is the limiting form of a perfect $n$-th power when $n$ is infinite.

Conversely, the only form for which $(a b)^{2}$ vanishes is the exponential function.

In fact, we have

$$
\begin{aligned}
& \left(\frac{\partial}{\partial x}-\frac{\partial}{\partial y}\right)^{2} f_{x} f_{y}=0 \quad(y=x), \\
& \text { i.e., } \\
& f \frac{d^{2} f}{d x^{2}}-\left(\frac{d f}{d x}\right)^{2}=0, \\
& f=C e^{a x}
\end{aligned}
$$


We next take the case of several exponential functions. The covariant $(b c)^{\lambda}(c a)^{\mu}(a b)^{\nu}$ of the symbolical forms $e^{\alpha x}, e^{\beta x}, e^{\gamma x}$ is

$$
(\beta-\gamma)^{\lambda}(\gamma-\alpha)^{\mu}(\alpha-\beta)^{\nu} e^{(a+\beta+\gamma) x},
$$

and for exponential forms a, $\beta, \gamma$ are actual numbers. Hence this covariant is a multiple of the product of the forms. This is obviously true in general.

Circular functions are next considered. If $f(x)=\phi(x)+\psi(x)$, it is obvious that

$$
(f f)^{2 \lambda}=(\phi \phi)^{2 \lambda}+2(\phi \psi)^{2 \lambda}+(\psi \psi)^{2 \lambda} .
$$

Hence, if

$$
\begin{gathered}
f(x)=A e^{m x}+B e^{n x}, \\
(a b)^{2}=(f f)^{2}=2 A B(m-n)^{2} e^{(m+n) x},
\end{gathered}
$$

and, in general,

$$
(a b)^{2 \lambda}=2 A B(m-n)^{2 \lambda} e^{(m+n) x} \text {. }
$$

If

$$
f(x) \equiv \cos x=\frac{e^{2 x}+e^{-i x}}{2}
$$

$$
(f f)^{2}=2 \cdot \frac{1}{2} \cdot \frac{1}{2}(2 \iota)^{2} e^{\iota x} e^{-\iota x}=-2
$$

and

$$
(f f)^{2 \lambda}=\frac{1}{2}(2 \iota)^{2 \lambda}=(-1)^{\lambda} 2^{2 \lambda-1} .
$$

Similarly, if $f(x)$ is $\sin x$,

Also

$$
(f f)^{2}=+2 \text { and }(f f)^{2 \lambda}=(-1)^{\lambda+1} 2^{2 \lambda-1} .
$$

$$
(a b)^{\lambda}(b c)^{\mu}=\left(\frac{\partial}{\partial x}-\frac{\partial}{\partial y}\right)^{\lambda}\left(\frac{\partial}{\partial y}-\frac{\partial}{\partial z}\right)^{\mu} f(x) f(y) f(z) \quad(y=z=x),
$$

and, if $f=\phi+\psi$,

$$
(a b)^{\lambda}(b c)^{\mu}=\left(\frac{\partial}{\partial x}-\frac{\partial}{\partial y}\right)^{\lambda}\left(\frac{\partial}{\partial y}-\frac{\partial}{\partial z}\right)^{\mu}\{\phi(x) \phi(y) \phi(z)+\phi(x) \phi(y) \psi(z)+\ldots\} .
$$

If $\phi(x)=A e^{m x}$ and $\psi(x)=B e^{n x}$, each term in the above expression vanishes identically except the terms obtained from $\phi(x) \psi(y) \phi(z)$ and $\psi(x) \phi(y) \psi(z)$. These give

$$
(-1)^{\mu} A^{2} B(m-n)^{\lambda+\mu} e^{(3 ! m+n) . x} \text { and }(-1)^{\lambda} A B^{2}(m-n)^{\lambda+\mu} e^{(2 n+m) x}
$$

respectively. Hence

$$
(a b)^{\lambda}(b c)^{\mu}=A B(n-n)^{\lambda+\mu}\left[(-1)^{\mu} A e^{m x}+(-1)^{\lambda} B e^{n x}\right] e^{(m+n) x},
$$

and we may show similarly that

$$
(b c)^{\lambda}(c a)^{\mu}(a b)^{\nu}=0 .
$$

If

$$
\begin{gathered}
f=\cos x=\frac{1}{2}\left(e^{a x}+e^{-\imath x}\right), \\
(a b)^{\lambda}(b c)^{\mu}=2^{\lambda+\mu-3} \iota^{(\lambda+\mu)}\left\lfloor(-1)^{\mu} e^{a x}+(-1)^{\lambda} e^{-\iota x}\right] .
\end{gathered}
$$


For example, $\quad(a b)^{2}(b c)=-\iota^{3} 2 \iota \sin x=-2 \sin x \quad$ if $f=\cos x$, and

$$
=2 \cos x \quad \text { if } f=\sin x .
$$

3. Before proceeding further with the calculation of covariants of particular forms, it will be convenient to obtain another expression for any covariant.

We consider

$$
\left(\frac{\partial}{\partial x}-\frac{\partial}{\partial y}\right)^{n} f(x) \phi(y) \quad(y=x) .
$$

If we write $x+h$ for $x$ and $y-h$ for $y$, this becomes

$$
\left(D_{1}+D_{2}\right)^{n} f(x+h) \phi(y-h) \quad(h=0 ; y=x),
$$

where $D_{1}=d / d h$ and operates only on $f$, whilst $D_{2}=d / d h$ and operates only on $\phi$.

But this is exactly $\left(\frac{d}{d h}\right)^{n} f(x+h) \phi(x-h) \quad(h=0)$,

or the covariant $(a b)^{n}$ of the two forms $f(x), \phi(x)$ is

$$
n !\left\{\text { coefficient of } h^{n} \text { in } f(x+h) \phi(x-h)\right\} .
$$

Again, take the expression

$$
\left(\frac{\partial}{\partial x}-\frac{\partial}{\partial y}\right)^{\lambda}\left(\frac{\partial}{\partial y}-\frac{\partial}{\partial z}\right)^{\mu} f(x) \phi(y) \psi(z) \quad(y=z=x) .
$$

This is

$$
\left(\frac{\partial}{\partial x}-\frac{\partial}{\partial y}\right)^{\lambda} f(x)\left(\frac{d}{d h}\right)^{\mu}[\phi(y+h) \psi(z-h)]
$$

$$
(h=0 ; y=z=x) \text {, }
$$

which becomes $\left(\frac{d}{d k}\right)^{\lambda} f(x+k)\left(\frac{d}{d h}\right)^{\mu}[\phi(y+h-k) \psi(z-h)] \quad\left(y^{\star}=z=x\right)$,

or

$$
\left(\frac{d}{d k}\right)^{\lambda}\left(\frac{d}{d h}\right)^{\mu} f(x+k) \phi(x+h-k) \psi(x-h) \quad(h=k=0)
$$

Hence the expression is equal to

$$
\lambda ! \mu !\left\{\text { coefficient of } k^{\lambda} h^{\mu} \text { in } f(x+k) \phi(x+h-k) \psi(x-h)\right\},
$$

and, in general, it is seen that a covariant may be expressed as a certain coefficient in the expansion of a function which may be immediately written down. As an example, consider the covariants of the Weierstrassian $\sigma$ function. Those of the type $(a b)^{\lambda}$ are the coefficients of the various powers of $h$ in the expansion of $\sigma(x+h) \sigma(x-h)$, each multiplied by an appropriate numerical factor.

Now

$$
\wp(x)-\wp(h)=-\frac{\sigma(x+h) \sigma(x-h)}{\sigma^{2}(x) \sigma^{2}(h)} .
$$


Hence $\quad \sigma(x+h) \sigma(x-h)=\sigma^{2}(h) \wp(h) \sigma^{2}(x)-\sigma^{2}(h) \rho(x) \sigma^{2}(x)$.

The covariants required are therefore of the type $\sigma^{2}(x)[A+B p(x)]$, where $A$ and $B$ are certain constants, $A$ being the coefficient of $h^{\lambda}$ in $\sigma^{2}(h) s h$, and $B$ being the coefficient of $h^{\lambda}$ in $-\sigma^{2}(h)$. This may also be written in the form $C \sigma(x+a) \sigma(x-a)$, where $C$ and $\alpha$ are constants.

In particular, if $\lambda=2$, the covariant is practically $\sigma^{2}(x) \&(x)$, whilst, if $\lambda=4$, it is $g_{2} \sigma^{2}(x) \times$ a numerical factor.

The covariants of the type $(a b)^{\lambda}(b c)^{\mu}$ are the various coefficients in the expansion of $\sigma(x+h) \sigma(x+k-h) \sigma(x-k)$.

Now $\frac{\sigma(x+h) \sigma(x+k-h) \sigma(x-k)}{\sigma^{3}(x)}$ is an elliptic function, since

$$
h+(k-h)+(-k)=0 .
$$

It is easily seen that it is $A p(x)+B \wp^{\prime}(x)+C$, where $A, B, C$ depend on $h$ and $k$ alone, and are readily determined. The covariants of this type are therefore $\left(a p x+b \wp^{\prime} x+c\right) \sigma^{3}(x)$, where $a, b, c$ are constants. These results admit of immediate generalization; for we are always concerned with a product of $n \sigma^{\prime}$ s, $\sigma(x+\alpha) \sigma(x+\beta) \ldots \sigma(x+\lambda)$, such that $\alpha+\beta+\ldots+\lambda=0$.

\section{Hence}

$$
\frac{\sigma(x+a) \sigma(x+\beta) \ldots \sigma(x+\lambda)}{\sigma^{n}(x)}
$$

is an elliptic function, and it has one irreducible infinity of the $n$-th order at the origin.

Hence it is $A+B \wp(x)+C \wp^{\prime}(x)+D \varphi^{\prime \prime}(x)+\ldots+K \boldsymbol{p}^{(n-2)}(x)$, where $A, B, C, \ldots, K$ are functions of $\alpha, \beta, \ldots$ only. The corresponding covariant is therefore

$$
\left[a+b \&(x)+c \wp^{\prime}(x)+\ldots+k \wp^{(n-2)}(x)\right] \sigma^{n}(x),
$$

where $a, b, \ldots, k$ are constants.

The covariants for the product of any number of $\sigma$ 's may be obtained in exactly the same manner.

4. The above is sufficient to show how the covariants of any power series may be calculated. We shall now proceed to find those functions for which the simpler covariants vanish. The corresponding problem for forms of finite order has often been dealt with by Hilbert and others; in the present case the only justification is the curious character of the results. It will be seen, in fact, that the first class, like

$$
(a b)^{2}, \quad(a b)^{2}(b c),
$$


lead to exponential functions, and the second class,

lead to elliptic functions.

$$
(a b)^{4}, \quad(a b)^{4}(b c)
$$

5. In solving the differential equation

$$
(a b)^{4}=0,
$$

which is

$$
\left(\frac{\partial}{\partial x}-\frac{\partial}{\partial y}\right)^{4} f_{x} f_{y}=0 \quad(y=x),
$$

or

$$
f f_{4}-4 f_{1} f_{3}+3 f_{2}^{2}=0,
$$

it is convenient to put $f=e^{u}$.

Now, in virtue of the foregoing, $(a b)^{4}$ is practically the coefficient of $h^{4}$ in the expansion of $f(x+h), f(x-h)$. Hence we want the coefficient of $h^{4}$ in

$$
e^{u+h u_{1}+\left(h n^{2} ; 21\right) u_{2} \ldots} e^{n-h u_{1}+\left(h^{2}, 2 !\right) u_{2} \ldots}=f^{2}\left\{1+\frac{A_{2}}{2 !} h^{2}+\frac{A_{4}}{4 !} h^{4} \ldots\right\},
$$

where

$$
1+\frac{A_{2}}{2 !} h^{2}+\frac{A_{4}}{4 !} h^{4} \ldots=e^{2\left(u_{2} h^{2} / 2 !+u_{4} h^{2} / 4 ! \ldots\right)}
$$

whence $A_{2}=2 u_{2}, \quad A_{4}=2\left(u_{4}+6 u_{2}^{2}\right), \quad A_{6}=2\left(u_{6}+30 u_{2} u_{4}+60 u_{2}^{3}\right), \quad \ldots$

6. Hence, if $(a b)^{2}=0$, we have

as before.

$$
u_{2}=0, \quad u=a x+b, \quad \text { and } \quad f=e^{(a x+b)},
$$

$$
\text { If }(a b)^{4}=0, \quad u_{4}+6 u_{2}^{2}=0,
$$

and, putting $u_{2}=w$, this is $w_{2}+6 w^{2}=0$.

$$
\text { Hence } \quad w=s\left\{c(x+c), 0, g_{3}\right\} \text {, }
$$

$c$ and $g_{3}$ being arbitrary constants.

Finally,

for

$$
f=e^{a x+b} \sigma\left\{x+c, 0, g_{3}\right\}
$$$$
w=+\frac{\partial^{2}}{\partial x^{2}}\{\log f\} \text {. }
$$

The equation $(a b)^{6}=0$ leads to new functions, and I have not yet studied it completely. It may be mentioned that the subsidiary equation

$$
w_{4}+30 w w_{2}+60 w^{3}=0
$$


admits a solution of the form

$$
\wp\left(\iota x+a, 0, g_{3}\right)-\wp\left(x+\beta, 0, g_{3}\right),
$$

$\alpha, \beta, g_{3}$ being arbitrary constants; further, any non-essential singularity of $w$ at a tinite distance is a pole of the second order. It seems extremely probable that any equation of the form

$$
(a b)^{2 \lambda}=0
$$

is satisfied only by integral functions.

7. The next form in order of simplicity is $(a b)^{\lambda}(b c)$.

Except for a numerical multiplier this is the coefficient of $h^{\wedge} k$ in

$$
f(x+h) f(x+k-h) f(x-k),
$$

which is that of $h^{\lambda}$ in

$$
f(x+h) f^{\prime}(x-h) f(x)-f(x+h) f(x-h) f^{\prime}(x) ;
$$

or, on putting $f=e^{u}$,

$$
\begin{aligned}
& e^{u+h u_{1}+\left(h^{2} / 2 !\right) u_{2} \ldots} e^{u-h u_{1}+\left(h^{2}, 2 !\right) u_{2} \ldots} e^{u}\left(u_{1}-h u_{2}+\frac{h^{2}}{2} u_{3}-\ldots\right) \\
& -e^{u+h u_{1}+\left(h^{2} / 21\right) u_{2} \ldots} e^{u-h u_{1}+\left(h^{2} / 2 !\right)} u_{2} \ldots e^{u} u_{1} \\
& \quad=f^{3} e^{2\left[\left(h^{2} \cdot 2 !\right) u_{2}+\left(h^{4}: 41\right) u_{4} \ldots\right]}\left(-h u_{2}+\frac{h^{2}}{2 !} u_{8}-\frac{h^{4}}{3 !} u_{4}+\ldots\right) .
\end{aligned}
$$

Writing this

$$
f^{3}\left(B_{1} h+\frac{B_{2} h^{2}}{3 !}+\frac{B_{8} h^{3}}{3 !}-\ldots\right)
$$

we have

$$
B_{1}=-u_{2}, \quad B_{2}=u_{3}, \quad B_{8}=-\left(u_{4}+6 u_{2}^{2}\right), \quad B_{1}=u_{5}+12 u_{2} u_{3} .
$$

It is not difficult to prove the general formulæ

$$
2 B_{2 n}=\frac{d}{d x} A_{2 n}, \quad 2 B_{2 n+1}=-A_{2 n+2},
$$

but they are useless for our present purposes, except as showing, for example, that the equations

$$
(a b)^{\lambda}=0, \quad(a b)^{\lambda}(b c)=0
$$

lead to similar functions. 
Returning to the equations, we see that $(a b)^{\lambda}(b c)=0$ leads to nothing new if $\lambda$ be odd, as is indeed a priori obvious.

Further,

$$
\begin{gathered}
(a b)^{2}(b c)=0 \text { gives } f=e^{a x^{3}+2 b x+c}, \\
(a b)^{4}(b c)=0 \text { gives } u_{4}+6 u_{2}^{2}=\text { const., } \\
f=e^{u}=e^{a x+b} \sigma\left\{x+c, g_{2}, g_{3}\right\}
\end{gathered}
$$

and thence

so the general $\sigma$ function is obtained.

Other examples could be easily discussed, e.g.,

$$
\begin{array}{lll}
(a b)^{2}(b c)^{2}(c a)^{2}=0 & \text { gives } f=e^{a x+b}+e^{c x+d}, \\
(a b)^{2}(b c)^{2}(c d)^{2}=0 & \text { gives } f=e^{a x+b} \sigma\left\{x+\alpha, g_{2}, 0\right\} .
\end{array}
$$

I propose to return subsequently to the interesting equation

$$
(a b)^{6}=0 \text {. }
$$

